\title{
Associative electrolyte solution near the charge hard wall. Density, charge, polarization and potential profiles
}

\author{
V.I.Kapko, M.F.Holovko \\ Institute for Condensed Matter Physics \\ of the National Academy of Sciences of Ukraine, \\ 1 Svientsitskii Str., 79011 Lviv, Ukraine
}

Received August 26, 2000

The density, charge, polarization and potential profiles of a simple model of an associative electrolyte are studied in an associative mean spherical approximation (AMSA). The limits of the full association and complete disassociation are considered.

Key words: electrolyte-electrode interface, associative mean spherical approximation

PACS: $05.20 .-y$

A set of important results for the electrode-electrolyte interface description can be obtained on the basis of models and methods, which have been developed and tested previously for bulk systems with electrostatic interactions and then generalized for inhomogeneous systems. The Henderson-Abraham-Barker (HAB) approach [1] enables us to describe the interface properties through the bulk properties of fluids. The simplest ion-dipole model for an electrolyte in the mean spherical approximation (MSA) [2] has been used to investigate the electrolyte properties near the charged wall by Blum and Henderson [3]. Recently the associative mean spherical approximation (AMSA) was solved [4], within the frameworks of which the analytical solution of the ion-dipole mixture against the charge hard wall has been obtained [5]. The purpose of this note is to discuss the influence of interionic association on the density, charge, polarization and potential profiles.

Our system consists of a mixture of charged hard spheres and dipolar hard spheres near the charged hard wall. Let us call $\rho_{+}, \rho_{-}, \rho_{\mathrm{d}}\left(\rho_{+}=\rho_{-}=\rho_{\mathrm{i}} / 2\right)$ the number density of the cations, anions and solvent, respectively. The diameters of all particles are equal to $\sigma$. The modules of the charges of the cations and anions are also equal $\left(Z_{+} e=-Z_{-} e=\mathrm{q}\right)$. The oppositely charged ions can form the neutral pairs due to the attractive sites placed on the surface of each ion.

The associative version of the wall-particle Ornstein-Zernike (OZ) equation can 
be written as

$$
h_{y}^{\beta}\left(z, \Omega_{2}\right)=c_{y}^{\beta}\left(z, \Omega_{2}\right)+\sum_{x} \sum_{\gamma \delta} \int \mathrm{d} 3 h_{x}^{\gamma}\left(z^{\prime}, \Omega_{3}\right) \rho_{x}^{\gamma \delta} C_{x y}^{\delta \beta}(32)
$$

together with MSA closure relations

$$
\begin{array}{r}
h_{y}^{\beta}\left(z, \Omega_{2}\right)=-\delta_{\beta 0}, \quad r_{12}<\sigma / 2 \\
c_{y}^{\beta}\left(z, \Omega_{2}\right)=-\delta_{\beta 0} \frac{u_{y}^{\mathrm{el}}(z)}{k T}, \quad r_{12} \geqslant \sigma / 2,
\end{array}
$$

where $h_{y}^{\beta}\left(z, \Omega_{2}\right), c_{y}^{\beta}\left(z, \Omega_{2}\right)$ are the vectors of the pair and direct wall-particle correlation functions, $z$ is the distance of the particle from the wall surface, $\Omega_{2}$ orientation of dipolar moment. Subscripts $x$ and $y$ point at the sort of the particle and superscripts $\alpha, \beta, \gamma$ and $\delta$ at the degree of bonding ( 0 for bonded and 1 for unbonded particle). $C_{x y}^{\alpha \beta}(12)$ is the matrix of the direct correlation functions for the bulk phase. d3 means integration over the positions $\vec{r}_{3}$ and possible orientations of the dipole.

The wall particle interaction is for the ion

$$
u_{\mathrm{i}}^{\mathrm{el}}(z)=-e E z
$$

and for the dipole

$$
u_{\mathrm{s}}^{\mathrm{el}}(z)=-(\vec{\mu} \vec{E})
$$

where $\vec{E}$ is the bare (unscreened) electric field, which is connected with the surface charge density on the wall $q_{\mathrm{s}}$ by

$$
E=4 \pi q_{\mathrm{s}} .
$$

Matrix of density is defined as $[6,7] \rho_{x}^{00}=\rho_{x}, \rho_{x}^{01}=\rho_{x}^{10}=\rho_{x}^{0}, \rho_{x}^{11}=0$, where $\rho_{x}$ is the total density of particles of sort $x$ and $\rho_{x}^{0}$ is density of unbonded particles of sort $x$, which are connected by self-consistent relation [8]:

$$
\rho_{\mathrm{i}}=\rho_{\mathrm{i}}^{0}+2 \pi\left(\rho_{\mathrm{i}}^{0}\right)^{2} \sigma^{3} B g_{+-}^{00}(\sigma+) .
$$

The analytical solution can be represented in the form of the two integral equations:

$$
\begin{aligned}
g_{y}^{(S) \beta}(z)-\sum_{x} \sum_{\gamma \delta} \rho_{x}^{*} \int_{0}^{z} g_{x}^{(S) \gamma}(z-r) R_{x}^{\gamma \delta} Q_{x y}^{(S) \delta \beta}(r) \mathrm{d} r & =K_{y}^{\beta}(0), \\
\sqrt{\rho_{y}^{*}} h_{y}^{(D) \beta}(z)-\sum_{x} \sum_{\gamma \delta} \sqrt{\rho_{x}^{*}} \int_{0}^{z} h_{x}^{(D) \gamma}(z-r) R_{x}^{\gamma \delta} Q_{x y}^{(D) \delta \beta}(r) \mathrm{d} r & =-F_{y}^{\beta}(z) .
\end{aligned}
$$

The expressions for $\hat{Q}^{(S)}(r), \hat{Q}^{(D)}(r), K_{y}^{\beta}(0)$ and $F_{y}^{\beta}(z)$ can be found in [5]. The density profiles are connected with the functions $g_{y}^{(S) \beta}(z)$ and $h_{y}^{(D) \beta}(z)$ by

$$
\begin{aligned}
g_{ \pm}(z) & =\sum_{\beta=0}^{1}\left(g_{\mathrm{i}}^{(S) \beta}(z) \pm h_{\mathrm{i}}^{(D) \beta}(z)\right) \\
g_{\mathrm{d}}(z, \Theta) & =g_{\mathrm{d}}^{(S) 0}(z)+\sqrt{3} h_{\mathrm{d}}^{(D) 0}(z) \cos (\Theta)
\end{aligned}
$$


with $\Theta$ the angle between the dipolar moment and the normal to the wall. It must be pointed out that the factor $\rho_{\mathrm{i}}^{0} / \rho_{\mathrm{i}}$ is included in $g_{\mathrm{i}}^{(S) 1}(z)$ and $h_{\mathrm{i}}^{(D) 1}(z)$ in contrast to Wertheim's definition $[6,7]$. This avoids the uncertainty in the case of full association $\left(\rho_{\mathrm{i}}^{0}=0\right)$.

We solve numerically the integral equations (7) and (8) by the well-known Perram's method [9].

The profiles in figures $1-4,7-8$ have been calculated by parameters

$$
\begin{aligned}
\eta & =\pi / 6 \times\left(\rho_{\mathrm{i}} \sigma^{3}+\rho_{\mathrm{d}} \sigma^{3}\right)=0.3178, \\
c_{\mathrm{i}} & =\rho_{\mathrm{i}} /\left(\rho_{\mathrm{i}}+\rho_{\mathrm{d}}\right)=0.0115, \\
q^{* 2} & =\frac{Z_{\mathrm{i}}^{2} e^{2}}{\sigma k T}=40, \\
\mu^{* 2} & =\frac{\mu^{2}}{\sigma^{3} k T}=2.5, \\
E^{*} & =\sqrt{\frac{\sigma^{3}}{k T} E}=1.5,
\end{aligned}
$$

which correspond to the Debye screening region. The ionic densities in the curves plotted in the figures 4 and 5 are $c_{\mathrm{i}}=0.11$ and $c_{\mathrm{i}}=0.5$ which correspond the shortrange ionic ordering and the short density ordering near the wall, respectively. We consider the three cases of the ionic association in all figures: (a) corresponds to the case of the complete dissociation (the ionic monomer fraction $\alpha=\rho_{\mathrm{i}}^{0} / \rho_{\mathrm{i}}=1$ ); (b) corresponds to $\alpha=0.5$; (c) corresponds to the case of the fully ionic dimerization $\alpha=0$.

The charge profiles at the low ionic concentration are plotted in figure 4 . The charge profile at $\alpha=0.5$ is close to the one for the free ions. The curve for the fully dimerized ions is less than the charge profiles for (a) and (b) cases. At $z=1.5 \sigma$ the curve (c) has a sharp maximum which indicates an orthogonal configuration of the dimers.

Figure 5 corresponds to the middle ionic concentration $c_{\mathrm{i}}=0.11$. For the fully dimerized ions (c) the charge profile sign change is observed at the first maximum region. At $z<0.8 \sigma$ all the curves coincide. For bigger distances $(z>2 \sigma)$ - after the first minimum the charge profiles decay fast.

In figure 6 the charge profiles for the comparatively high concentration $c_{\mathrm{i}}=0.5$ are shown. Under such conditions the dimerization process exerts no influence on the charge profiles. At $z>1.5 \sigma$ we observe some lag between (c)-(b), (b)-(a), (c)-(a) profiles.

The polarization profiles (figure 7) possess the oscillative behaviour which corresponds to a solvent discrete structure. At high distances $(z>2.5 \sigma)$ the higher degree of the ionic association leads to the increase of a solvent polarization.

The potential profiles are plotted in figure 8. They also show the oscillative behaviour and decay at high z. The association leads to potential profiles increasing especially for low $\alpha$. 


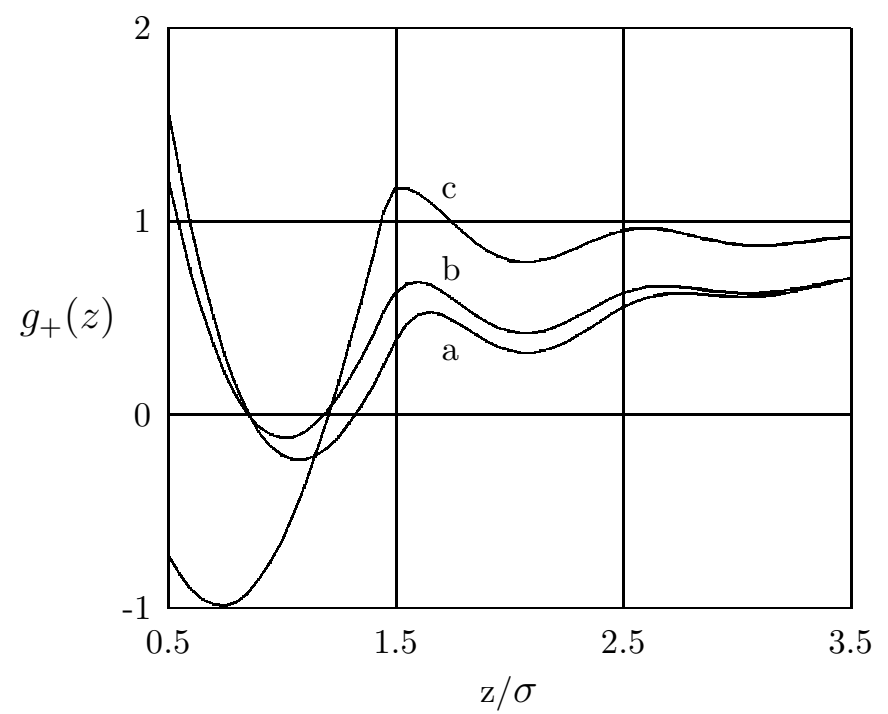

Figure 1. The density profiles of cations at $c_{\mathrm{i}}=0.0115$.

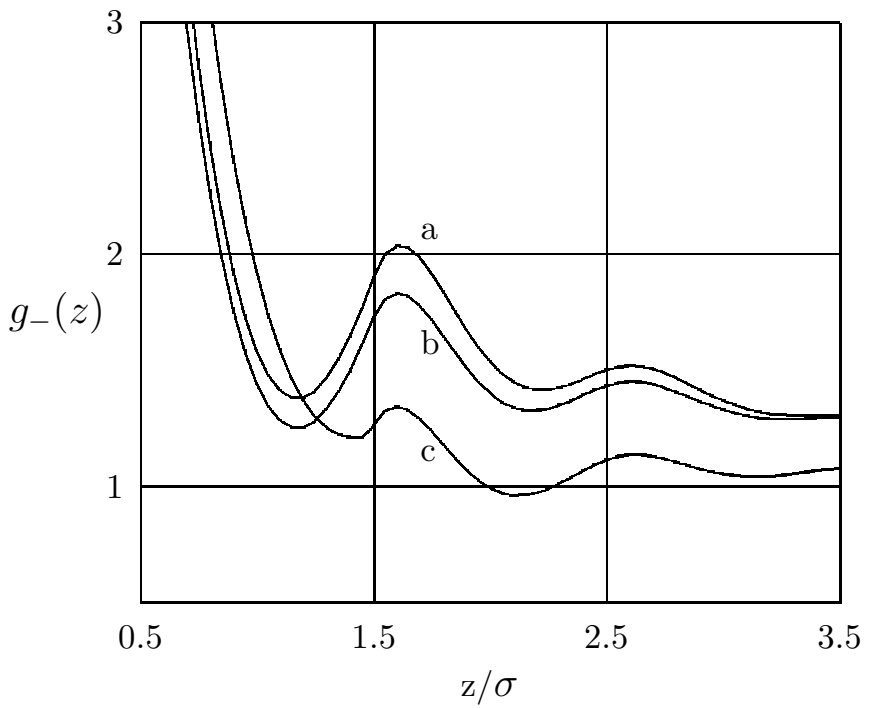

Figure 2. The density profiles of anions at $c_{\mathrm{i}}=0.0115$. 


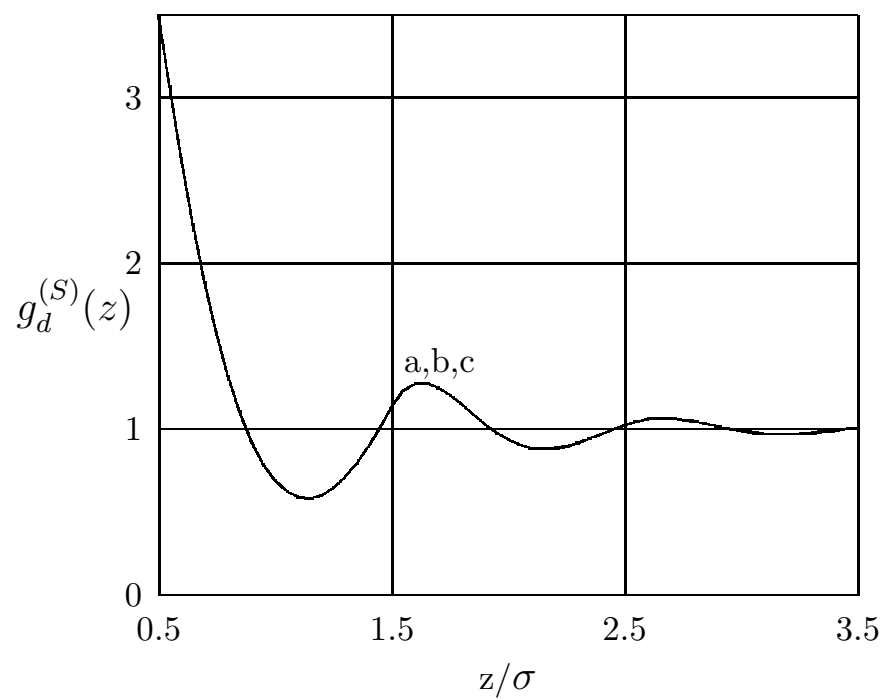

Figure 3. The S-part of solvent density profiles at $c_{\mathrm{i}}=0.0115$.

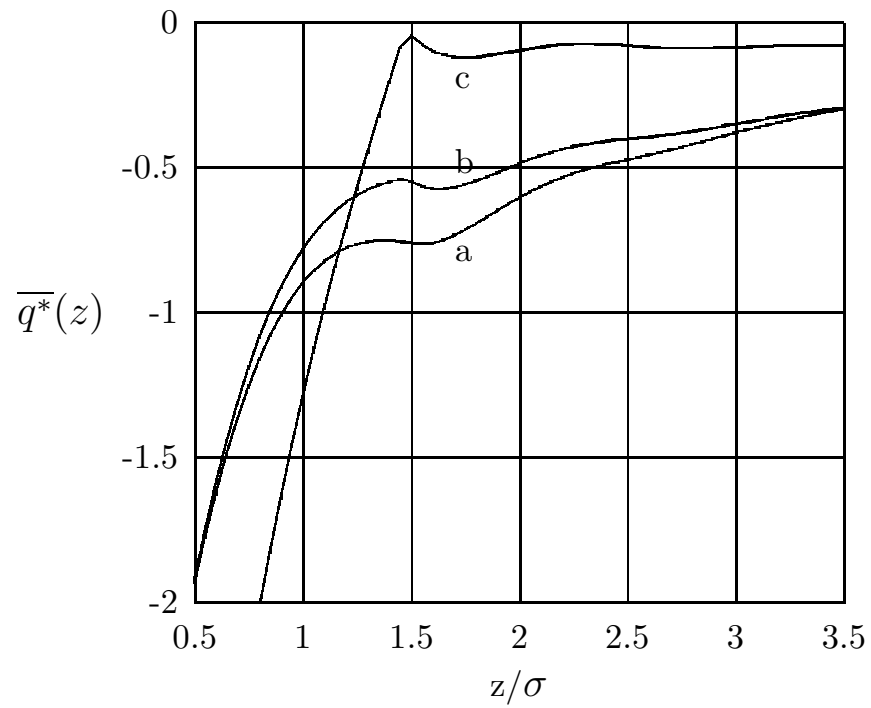

Figure 4. The charge profiles at $c_{\mathrm{i}}=0.0115$. 


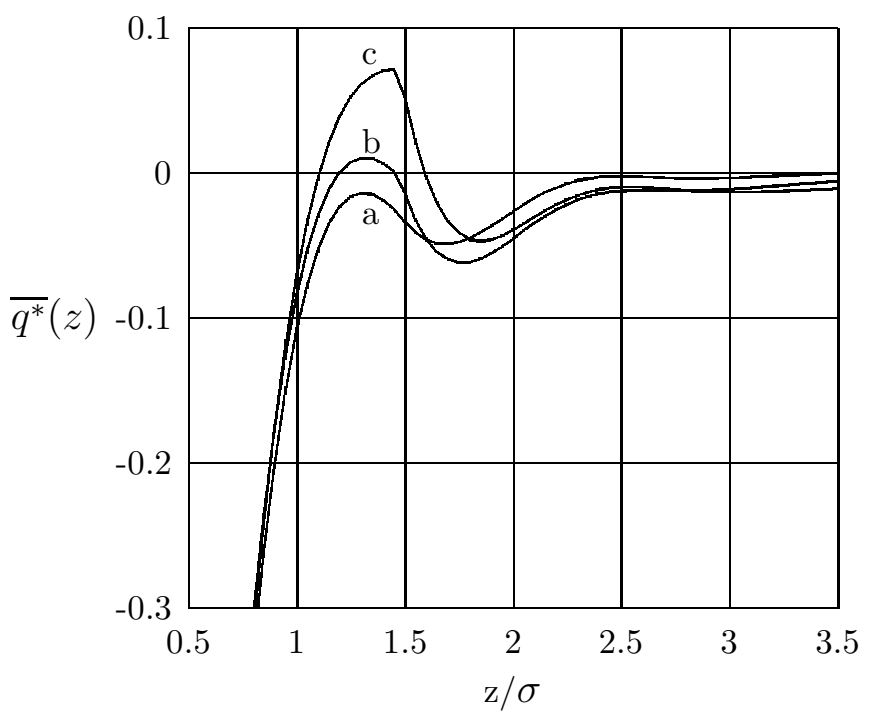

Figure 5. The charge profiles at $c_{\mathrm{i}}=0.11$.

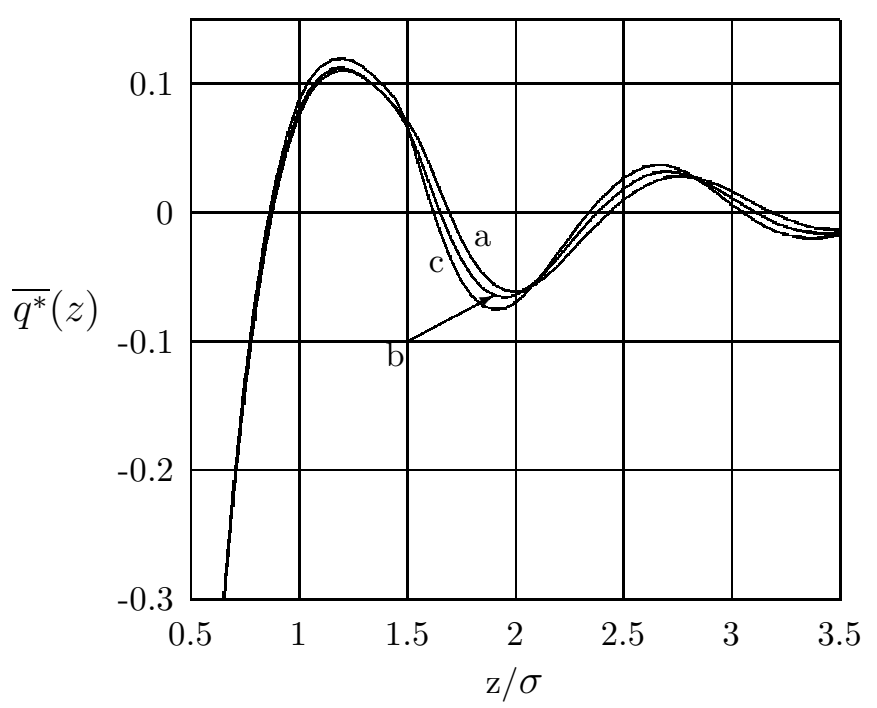

Figure 6. The charge profiles at $c_{\mathrm{i}}=0.5$. 


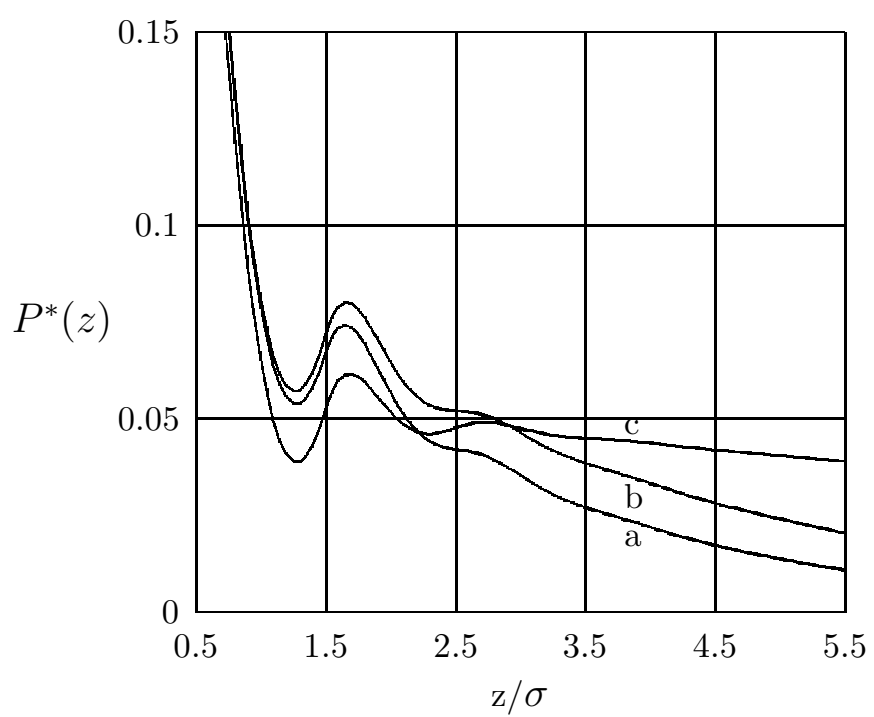

Figure 7. The polarization profiles at $c_{\mathrm{i}}=0.0115$.

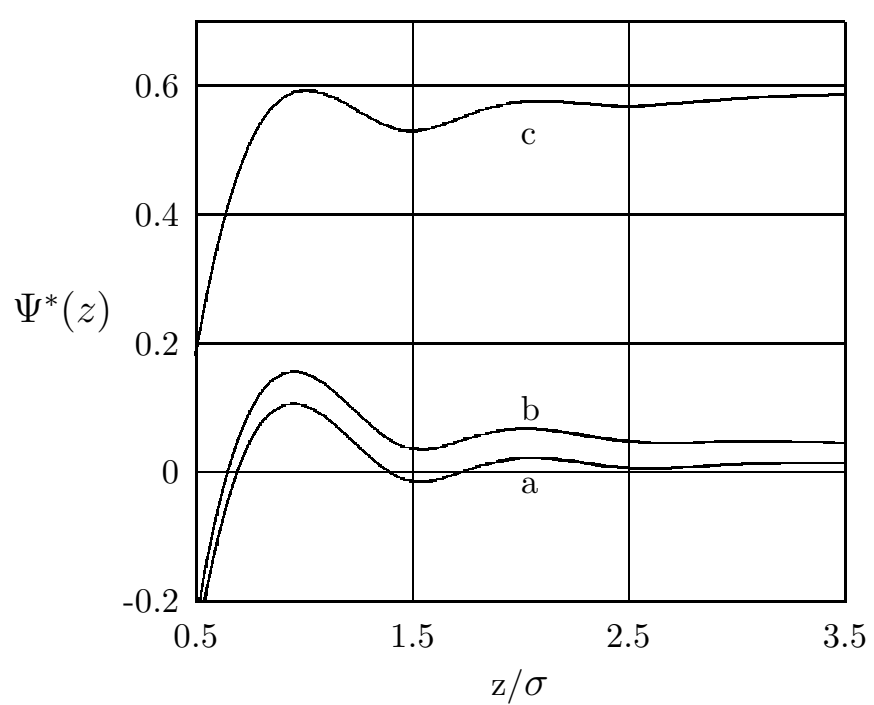

Figure 8. The potential profiles at $c_{\mathrm{i}}=0.0115$. 


\section{References}

1. Henderson D., Abraham F.F., Barker J.A. // Molec. Phys., 1976, vol. 31, p. 129.

2. Blum L. // J. Chem. Phys., 1974, vol. 61, p. 2129.

3. Blum L., Henderson D. // J. Chem. Phys., 1981, vol. 74, p. 1902.

4. Holovko M.F., Kapko V.I. // Condens. Matter Phys., 1998, vol. 1, p. 239.

5. Holovko M.F., Kapko V.I. (in preparation).

6. Wertheim M.S. // J. Stat. Phys., 1984, vol. 35, p. 19.

7. Wertheim M.S. // J. Stat. Phys., 1984, vol. 35, p. 35.

8. Holovko M.F., Kalyuzhnyi Yu.V. // Mol. Phys., 1991, vol. 73, p. 1145.

9. Perram J.W. // Mol. Phys., 1975, vol. 30, p. 1505.

\section{Асоціативний електроліт біля зарядженої твердої} стінки. Профілі густини, заряду, поляризації і потенціалу

\section{В.І.Капко, М.Ф.Головко}

Інститут фізики конденсованих систем НАН України, 79011 Львів, вул. Свєнціцького, 1

Отримано 26 серпня 2000 р.

Профілі густини, заряду, поляризації і потенціалу простої моделі асоціативного електроліту вивчаються в асоціативному середньо-сферичному наближенні (АСCH). Розглядаються границі повної асоціації і дисоціації.

Ключові слова: інтерфейс електроліт-електрод, асоціативне середньо-сферичне наближення

PACS: 05.20.-y 